\title{
A Sequential Procedure for Individual Identity Verification Using ECG
}

\author{
John M. Irvine ${ }^{1}$ and Steven A. Israel ${ }^{2}$ \\ ${ }^{1}$ Advanced Signal Processing and Image Exploitation Group, Draper Laboratory, 555 Technology Square, MS 15, \\ Cambridge, MA 02139, USA \\ ${ }^{2}$ Systems and Technology Division, SAIC, 4001 Fairfax Drive, Suite 450, Arlington, VA 22203, USA
}

Correspondence should be addressed to Steven A. Israel, steven.a.israel@saic.com

Received 20 October 2008; Revised 14 January 2009; Accepted 24 March 2009

Recommended by Kevin Bowyer

The electrocardiogram (ECG) is an emerging novel biometric for human identification. One challenge for the practical use of ECG as a biometric is minimizing the time needed to acquire user data. We present a methodology for identity verification that quantifies the minimum number of heartbeats required to authenticate an enrolled individual. The approach rests on the statistical theory of sequential procedures. The procedure extracts fiducial features from each heartbeat to compute the test statistics. Sampling of heartbeats continues until a decision is reached-either verifying that the acquired ECG matches the stored credentials of the individual or that the ECG clearly does not match the stored credentials for the declared identity. We present the mathematical formulation of the sequential procedure and illustrate the performance with measured data. The initial test was performed on a limited population, twenty-nine individuals. The sequential procedure arrives at the correct decision in fifteen heartbeats or fewer in all but one instance and in most cases the decision is reached with half as many heartbeats. Analysis of an additional 75 subjects measured under different conditions indicates similar performance. Issues of generalizing beyond the laboratory setting are discussed and several avenues for future investigation are identified.

Copyright (C) 2009 J. M. Irvine and S. A. Israel. This is an open access article distributed under the Creative Commons Attribution License, which permits unrestricted use, distribution, and reproduction in any medium, provided the original work is properly cited.

\section{Introduction}

The biometric verification process can be broken into five major functional blocks: data collection, signal processing, feature extraction, comparison (database lookup), and returning a decision (Figure 1). Verification systems have two competing requirements: (1) quickly processing samples and returning a decision to minimize the user time, and (2) operate at very high probability of detections (Pds) with low false alarm rates (FARs). With the advances in computing, the longest duration function in Figure 1's critical path is the data collection. This paper presents a method for quantifying the minimum number of heartbeats required for verifying the identity of an individual from the electrocardiogram (ECG) signal. The minimum number of heartbeats required provides a user-centric measure of performance for an identity verification system. The outcome of our research forms the basis for selecting elements of an operational ECG verification system.
Since 2001, researchers have identified unique characteristics of the ECG trace for biometric verification, particularly with respect to access control [1-14]. To illustrate, consider the heartbeats from several different individuals (Figure 2). Although each heartbeat follows the same general pattern, differences in the detailed shape of the heartbeat are evident. We exploit these shape differences across individuals to perform identity verification. The last 30 years have witnessed substantial research into the collection and processing of digital ECG signals [15-17]. In addition, a special issue of this journal was devoted to "Advances in electrocardiogram signal processing and analysis" in 2007 . We build on this wealth of information and apply it to the development of an ECG verification system.

Hawkins [18] revealed that the traditional biometrics of face, fingerprints, and iris can be forged. The traditional biometrics cited above contain no inherent measure of liveness. The ECG, however, is inherently an indication of liveness and, consequently, is difficult to falsify. Israel et al. 


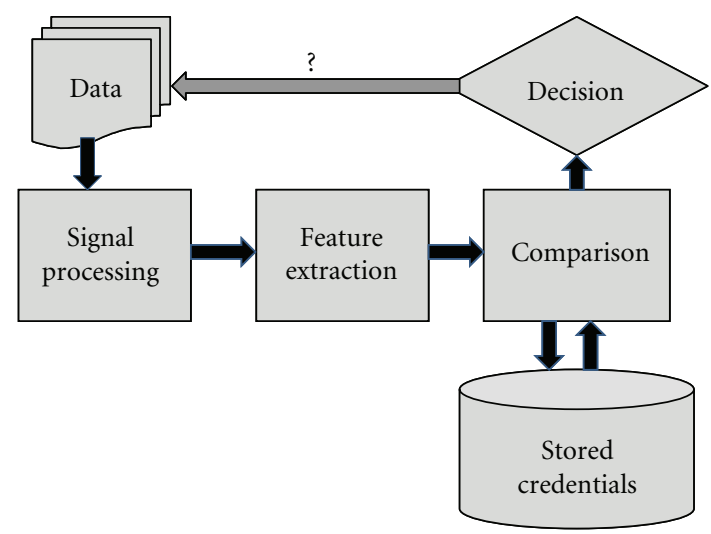

FIgURE 1: Simplified architecture for an authentication system.

[6] analyzed other cardiovascular modalities and found ECG data most discriminating for human identification.

This paper illustrates a methodology and minimum heartbeat performance metric using data and processing from our previously published research [1-7]. This work extends previous results in two ways. First, it focuses on the identity verification problem, such as would be appropriate for portal access. Second, the method developed here quantifies the minimum number of heartbeats needed for identity verification, thereby fixing the time needed to collect user data. The next section summarizes the utility of applying ECG information as a biometric. The following two sections present the actual methodology, first discussing the processing of the ECG signal and then deriving the actual test statistic used for identity verification. We present results from two data sets to illustrate performance. The final section discusses a number of practical issues related to ECG as a biometric and suggests avenues for further investigation.

\section{Background}

This paper presents a new approach for processing the ECG for identity verification based on sequential procedures. A major challenge for developing biometric systems based on circulatory function is the dynamic nature of the raw data. Heartrate varies with the subject's physical, mental, and emotional state, yet a robust biometric must be invariant across time and state of anxiety. The heartbeat maintains its structure with changes in heartrate (Figure 2). Irvine et al. $[1-3,5]$, Israel et al. $[4,6]$, and Biel et al. [8] identified individuals based upon features extracted from individual heartbeats. Wang et al. [9] followed a similar approach using fiducial features, but then extended the analysis based on a discrete cosine transform (DCT) of the autocorrelation function. Shen et al. [10] and Wubbeler et al. [14] employed a template matching approaches. Additional nonfiducial techniques have exploited principal components analysis (PCA) [19-27] in the same manner as [28] applied to face. Recently, a number of researchers have explored improvements to representations of the ECG signal for human identification $[5,9,29]$. In each case, the extracted ECG attributes performed well for identifying individuals.
Early studies of ECG feature extraction used spectral features to characterize the heartbeat [17]. Later, Biel et al. [8] performed ECG feature extraction by estimating location and magnitude information. Irvine et al. [2] showed that the relative electrode position caused changes in the magnitude of the ECG traces and used only temporal features. To these ends, Israel et al. [4] identified additional fiducial positions to characterize the relative intervals of the heartbeat and performed quantitative feature extraction using radius of curvature features.

Initial experiments for human identification from ECG identified some important challenges to overcome. First, approaches that rely on fiducial attributes, that is, features obtained by identifying specific landmarks from the processed signal have difficulty handling nonstandard heartbeats and high noise floors. Agrafioti and Hatzinakos [30] applied signal processing methods to address common cardiac irregularities. A second challenge is to insure that the identification procedure is robust to changes in the heartrate arising from varying mental and emotional states. Irvine et al. $[1-3]$ and Israel et al. $[4,6]$ addressed this issue through an experimental protocol that varied the tasks performed by the subjects during data collection. Third, PCA type algorithms must sample a sufficiently wide population to ensure the best generalization of their eigen features.

The ECG measures the electrical potential at the surface of the body as it relates to the activation of the heart. Many excellent references describe the functioning of the heart and the factors affecting the ECG signal $[15,31,32]$. Because the ECG consists of repeated heartbeats, the natural period of the signal is amenable to a wealth of techniques for statistical modeling. We exploit this periodic structure, treating the heartbeat as the basic sampling unit for constructing the sequential method.

\section{Signal Processing}

We segmented the data into two nonoverlapping, block segmented by time, groups. Group 1 is the training data, where labeled heartbeats are used to generate statistics about each enrolled individual. Group 2 is the test data, which contain heartbeats from the sensor and have known $a$ posteriori labels. The computational decision from the system is either a confirmation that the individual is who they say they are; or a rejection that the individual is not who they say they are.

Processing of the ECG signal includes noise reduction, segmentation of the heartbeats, and extraction of the features from each heartbeat (Figure 3). Because the objective is to minimize the data acquisition time for identity verification, the enrollment time was not constrained. Two minutes of data were used for enrollment and to train the verification functions for each individual. Two additional minutes of test data were available to quantify the required number of heartbeats. For our concept of operations, however, the individuals seeking authentication would only need to present the minimum number of heartbeats, which is expected to be on the order of second(s). 


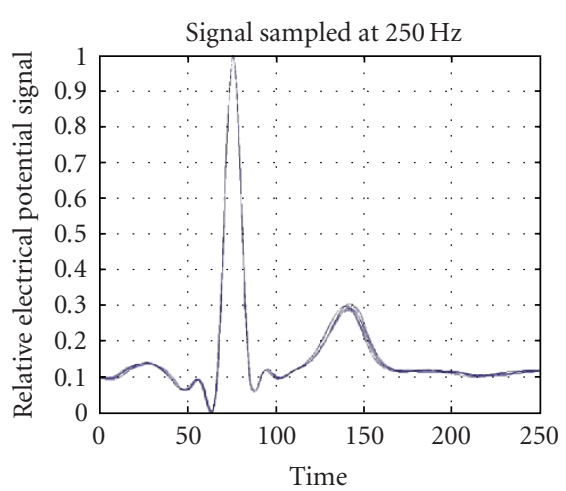

(a)

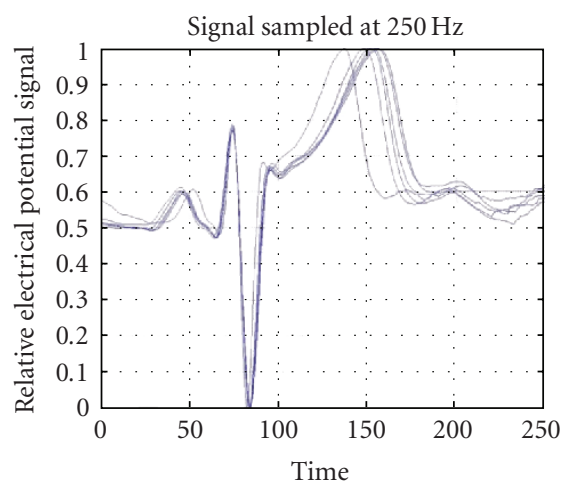

(d)

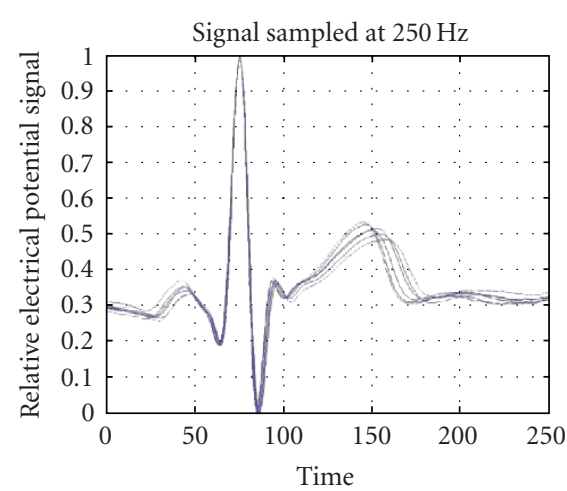

(b)

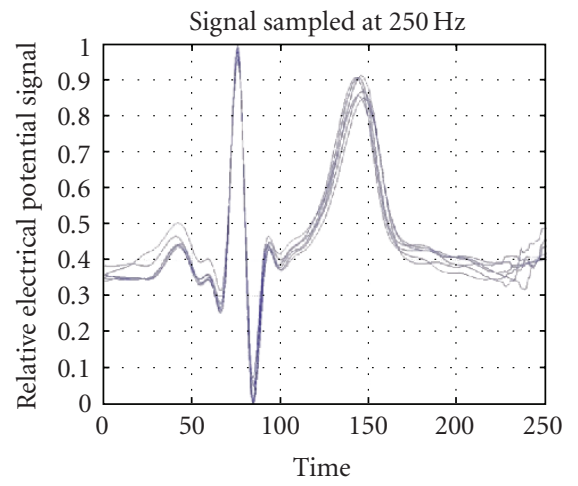

(e)

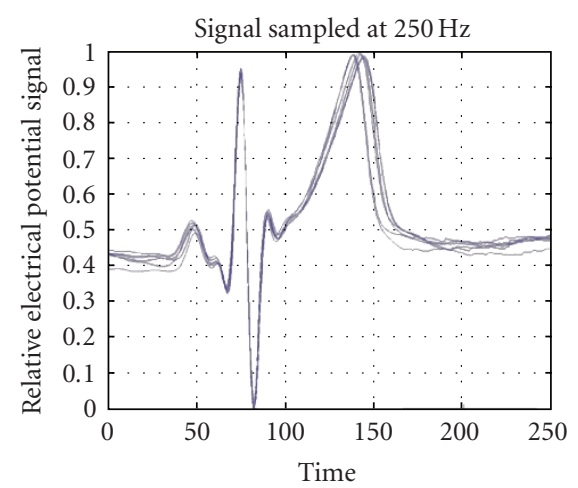

(c)

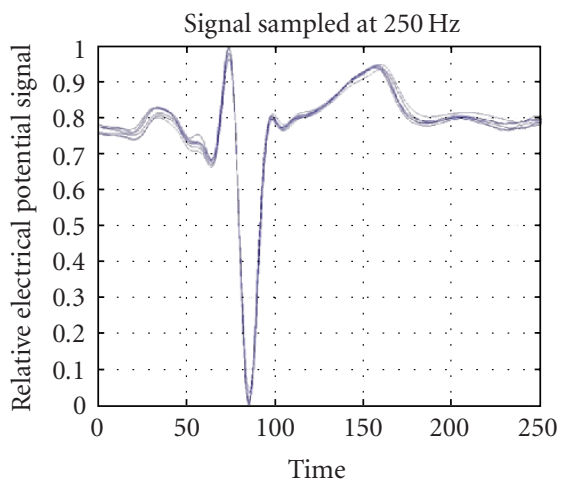

(f)

FIGURE 2: Segmented heartbeats from six individuals.
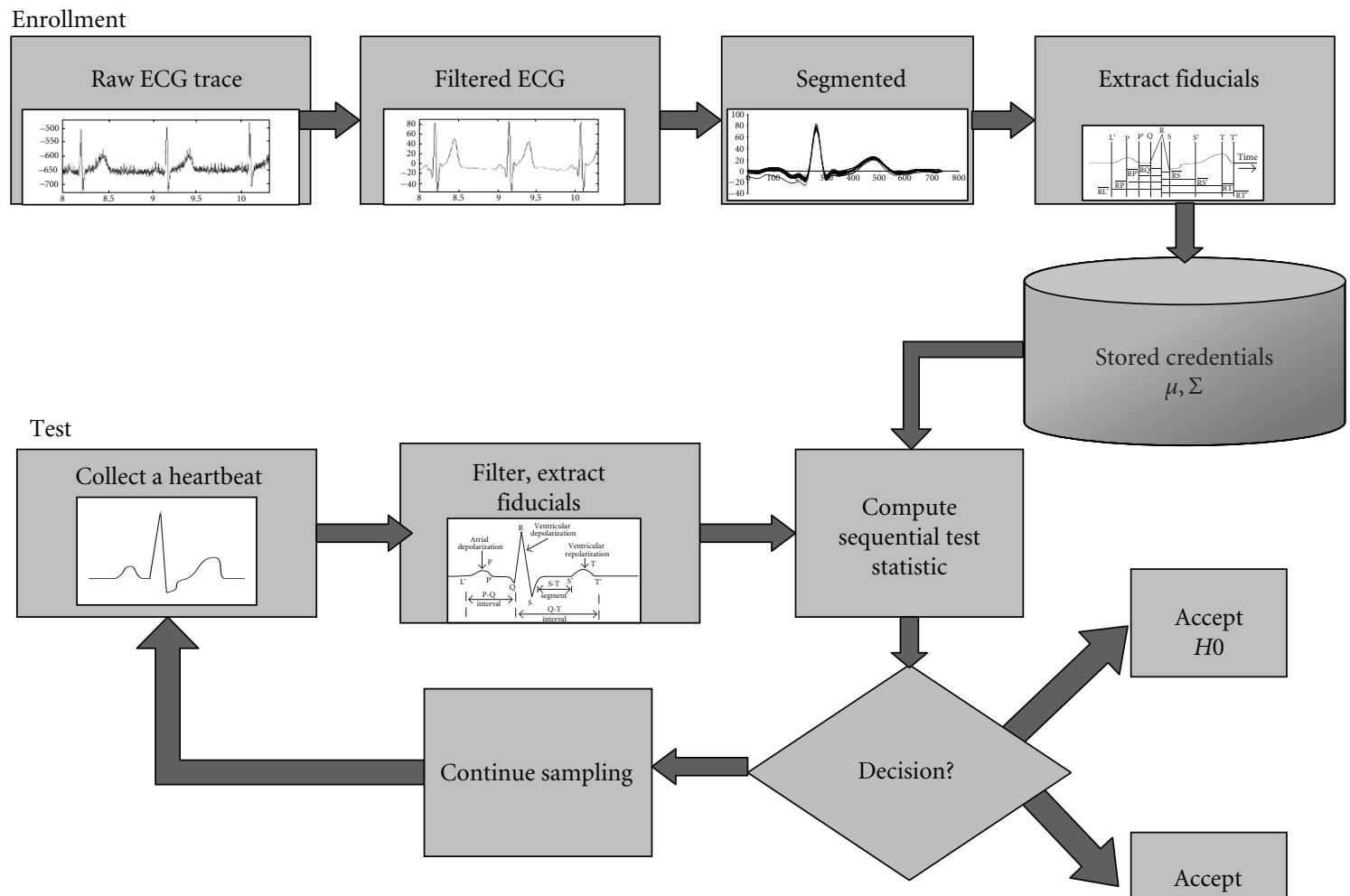

Accept

FIGURE 3: Signal processing for the sequential procedure. 


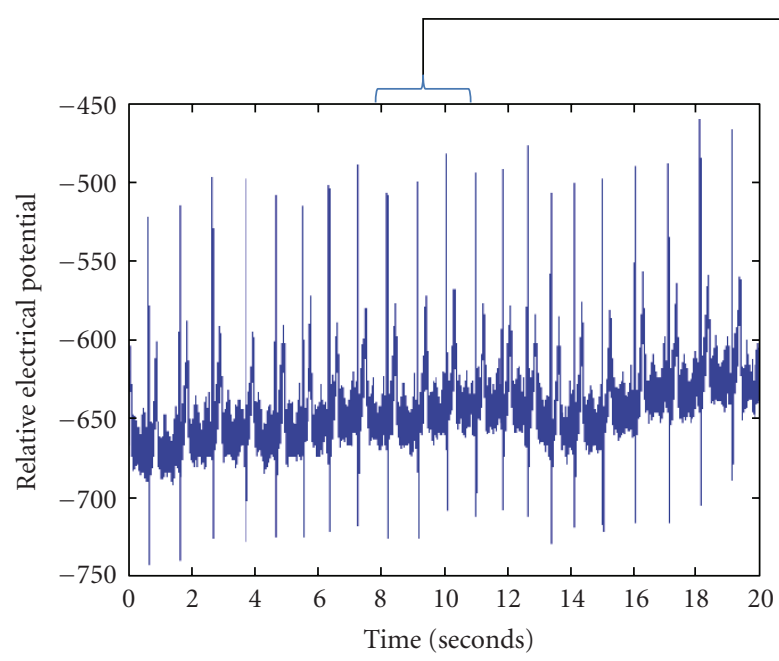

(a)

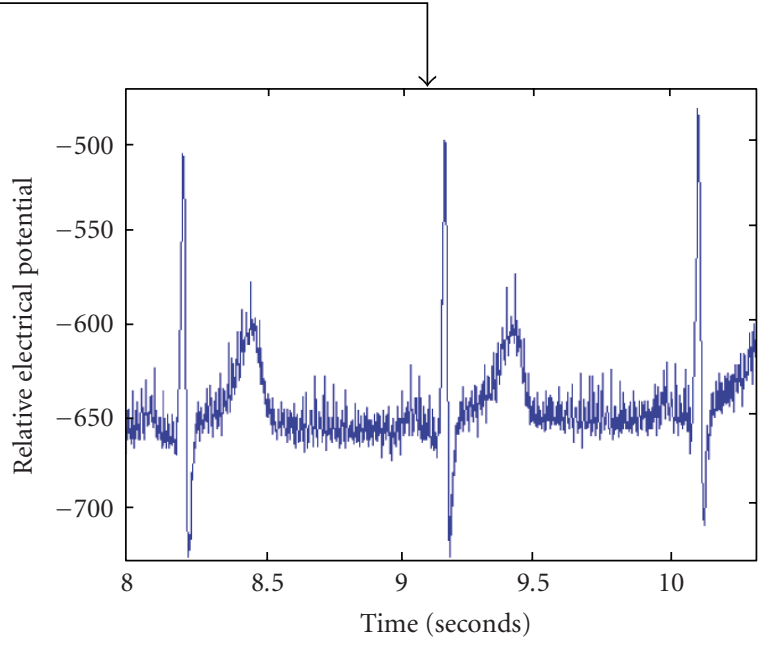

(b)

FIgUre 4: Raw ECG data $1000 \mathrm{~Hz}$ (a) 20 seconds (b) 2 seconds.

Figures 4(a) and 4(b) show a sample of high resolution ECG data. The raw data contain both high and low frequency noise components. These noise components alter the expression of the ECG trace from its ideal structure. The low frequency noise is expressed as the slope of the overall signal across multiple heartbeat traces in Figure 4(a). The low frequency noise is generally associated with changes in baseline electrical potential of the device and is slowly varying. Over this 20-second segment, the ECG can exhibit a slowly varying cyclical pattern, associated with respiration, that is known as sinus arrhythmia [15]. The high frequency noise is expressed as the intrabeat noise shown in Figure 4(b). The high frequency noise is associated with electric/magnetic field of the building power (electrical noise) and the digitization of the analog potential signal (A/D noise). Additionally, evidence of subject motion and muscle flexure must be removed from the raw traces [33].

Multiple filtering techniques have been applied to the raw ECG traces: heartbeat averaging $[34,35]$, wavelet [36, 37], least squares modeling [38, 39] and Fourier bandpass filtering [40-42]. For any filtering technique, the design constraints are to maintain as much of the subject-dependent information (signal) as possible and design a stable filter across all subjects.

As previously reported [4], the raw ECG traces were bandpass filtered between 0.2 and $40 \mathrm{~Hz}$. The filter was written with a lower order polynomial to reduce edge effects. Figure 5(a) illustrates the power spectra from a typical $1000 \mathrm{~Hz}$ ECG trace. The noise sources were identified, and our notional bandpass filter overlays the power spectrum. Figure 5(b) shows the power spectrum after the bandpass filtering. Figure 6 contains the processed data for heartbeat segmentation and feature extraction.

Commonly, heartbeat segmentation is performed by first locating the $R$ complex. Next, the $R$ position is estimated for the following heartbeats $[43,44]$. Our $R$ peak locator used a simple technique of looking at the maximum variance over a 0.2 second interval. The 0.2 -seconds represent ventricular depolarization. The metric was computed in overlapping windows to insure that the true $R$ peaks were recovered [4]. The remainder of the heartbeat was realized by locating the $P$ and the $T$ peaks relative to the $R$ position. For the enrollment data, we used autocorrelation techniques to develop an initial estimate of the $R-R$ interval. In the autocorrelation function, the lag for the maximum peak generally corresponds to the mean length of the heartbeat, giving an initial value to guide the heartbeat segmentation.

ECG data are commonly collected by contact sensors at multiple positions around the heart. The change in ECG electrode position provides different information because of the relative position to the heart's plane of zero potential. For nearly all individuals and all electrode locations, the ECG trace of a heartbeat produces three complexes (wave forms). The medical community has defined the complexes by their peaks: $P, R$, and $T$ (Figure 7). The $R-R$ interval, the time between two successive $R$ peaks, indicates the duration of a heartbeat. Two other fiducials, $Q$ and $S$, are also identified at the base of the $R$ complex. Israel et al. [4] identified four additional fiducials at the base of the $P$ and $T$ complexes. These are noted with a prime ( () symbol (Figure 7). We employ the single channel feature extraction method developed by Israel et al. [4]. The nine features derived from the fiducials are the feature vector used to illustrate the sequential procedure and the minimum number of heartbeats metric.

\section{The Sequential Procedure}

Abraham Wald developed the sequential procedure for formal statistical testing of hypotheses in situations where data can be collected incrementally $[45,46]$. In many instances, the sequential method arrives at a decision based on relatively 


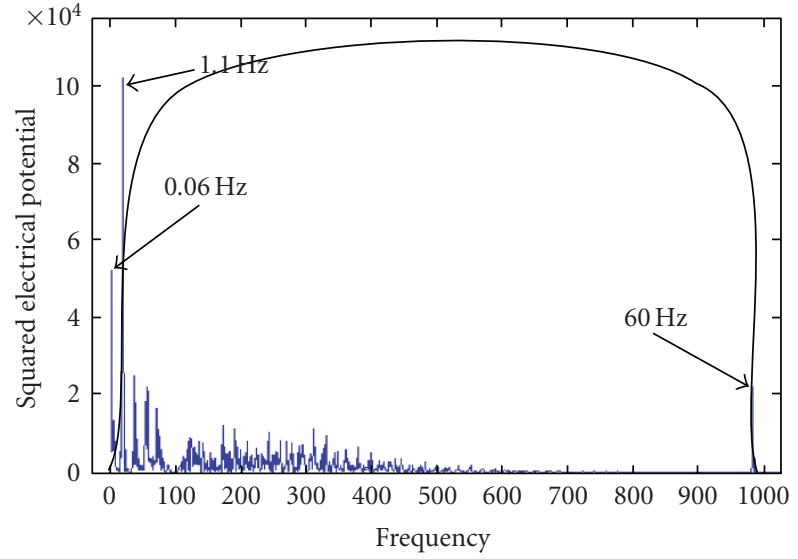

(a)

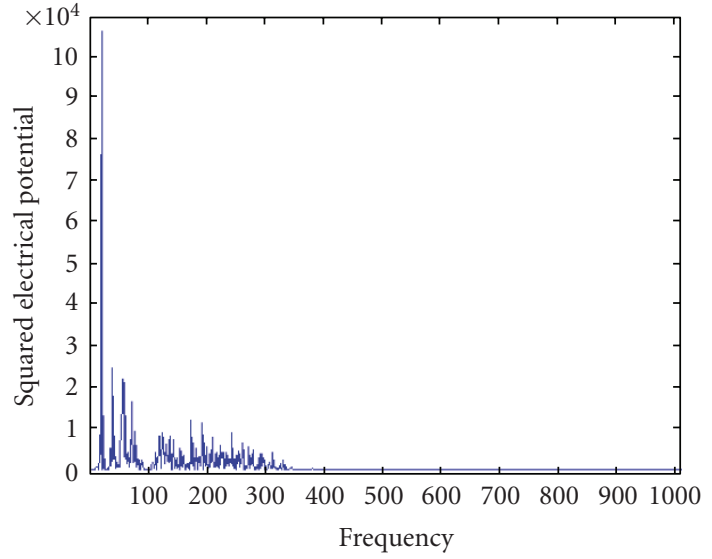

(b)

FIGURE 5: Power spectra of frequency filtering: (a) bandpass filter of raw data (b) frequency response of filtered data. (a) shows the noise source spikes at 0.06 and $60 \mathrm{~Hz}$ and the information spikes between 1.10 and $35 \mathrm{~Hz}$. (b) shows the filtered data with the noise spikes removed and the subject specific information sources retained. The $X$-axis is frequency in $\mathrm{Hz}$, and the $Y$-axis is squared electrical potential.

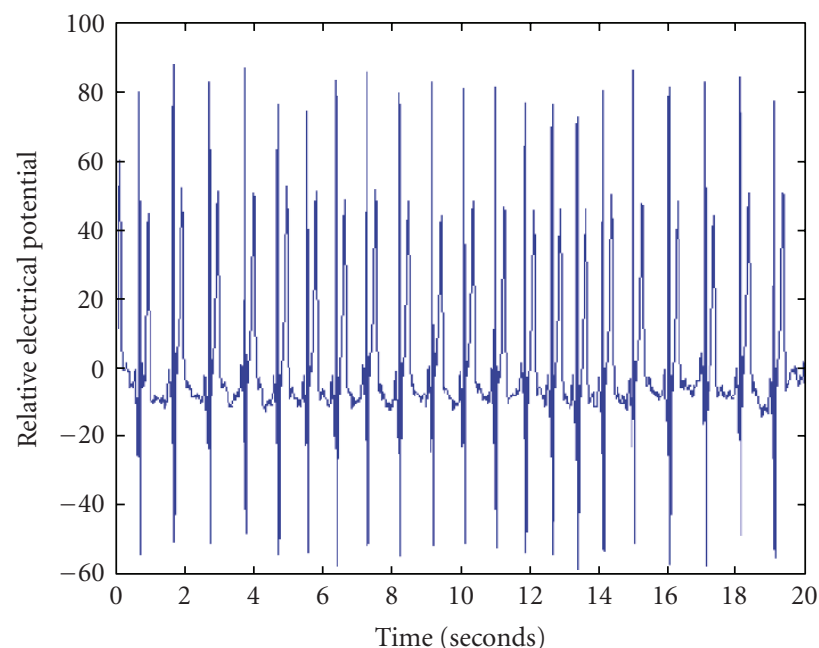

(a)

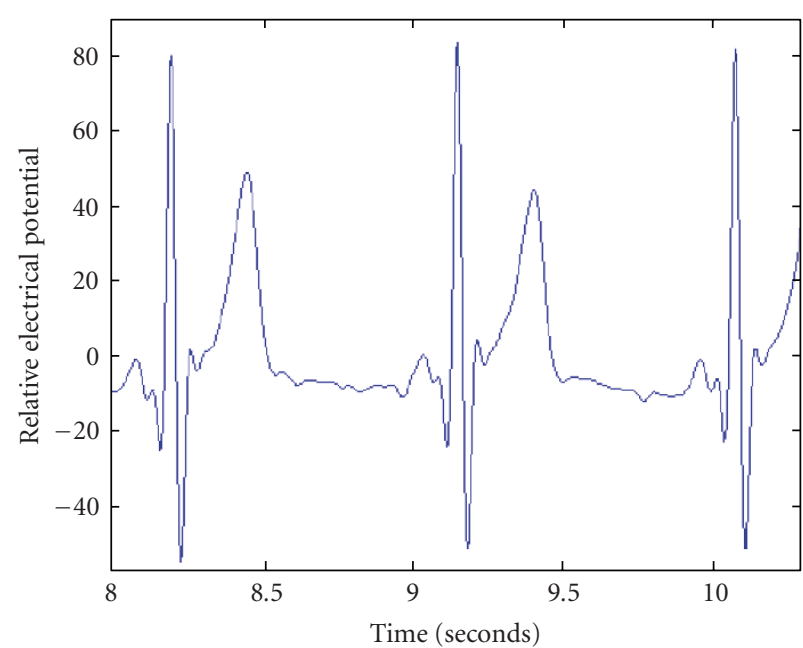

(b)

Figure 6: Bandpass filtered ECG trace (a) entire range of data (b) segment of data. The results of applying the filter (Figure 5) to the raw (Figure 4) data are shown.

few observations. Consider a sequence of independent and identically distributed random variables $\left\{X_{1}, X_{2}, \ldots\right\}$ and suppose we wish to test the hypothesis $H 0: X_{i} \sim f\left(X, \theta_{0}\right)$ against the alternative $H 1: X_{i} \sim f\left(X, \theta_{1}\right)$. The general approach is to construct the sequential probability ratio statistic for the first $T$ observations:

$$
S(T)=\frac{P\left[X_{1}, \ldots, X_{T} \mid H_{1}\right]}{P\left[X_{1}, \ldots, X_{T} \mid H_{0}\right]}=\frac{\prod_{t=1}^{T} f\left(X_{t}, \theta_{1}\right)}{\prod_{t=1}^{T} f\left(X_{t}, \theta_{0}\right)}
$$

At each step in the sequential procedure, that is, for each value of $T=1,2, \ldots$, the computed value of $S(T)$ is compared to the decision thresholds $A$ and $B$, where $0<A<$ $1<B<\infty$. The values of $A$ and $B$ depend on the acceptable level of error in the test of hypothesis. The decision procedure is

$$
\begin{aligned}
& \text { If } S(T)<A \text {, accept } H 0, \\
& \text { If } S(T)>B \text {, accept } H 1, \\
& \text { If } A<S(T)<B \text {, continue sampling, }
\end{aligned}
$$

$S(T)$ is known as the sequential probability ratio statistic. It is often convenient to formulate the procedure in terms of the log of the test statistic:

$$
\begin{aligned}
S^{*}(T) & =\log [S(T)] \\
& =\sum_{t=1}^{T} \log \left[f\left(X_{t}, \theta_{1}\right)\right]-\sum_{t=1}^{T} \log \left[f\left(X_{t}, \theta_{0}\right)\right] .
\end{aligned}
$$




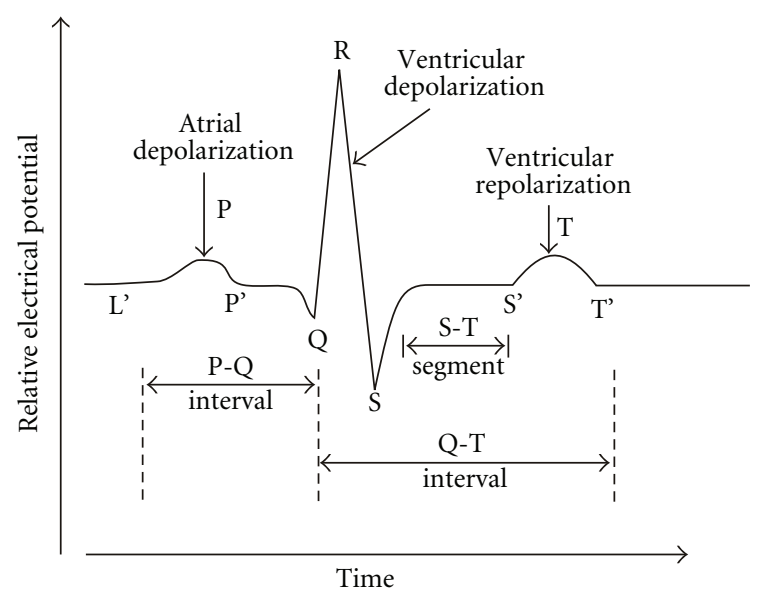

Figure 7: Fiducial features in the heartbeat.

To develop the sequential procedure for our application, we treat identity verification as a test of hypotheses. The two hypotheses are

$$
\begin{aligned}
& H 0: \text { The subject is who (s)he says } \\
& \text { H1: The subject is not who (s)he says. }
\end{aligned}
$$

The data for testing the hypotheses is the series of observed heartbeats presented in the test data. From each test heartbeat the fiducial features are extracted, forming a feature vector. Denote these feature vectors from each heartbeat by $\{H(1), H(2), \ldots\}$. If the person says (s)he is subject $i$, then $\{H(t): t=1, \ldots, T\}$ are drawn from the statistical distribution corresponding to subject $i$. If (s)he is not who (s)he claims to be, then $\{H(t): t=1, \ldots, T\}$ are drawn from a population with a statistical distribution corresponding to subject $j$, where $i \neq j$. To simplify the procedure, we assume that the feature vectors $\{H(t)\}$ are independent with a $K$ variate Gaussian distribution, where $K$ is the number of features extracted from each heartbeat. The mean vectors and covariance matrices are estimated from the enrollment data. Using this model for the test data, the hypotheses are restated in statistical terms:

$$
\begin{aligned}
& H 0: H(t) \sim \mathcal{N}\left(Y_{i}, \Sigma\right) \text { for }\{H(t): t=1, \ldots, T\}, \\
& H 1: H(t) \sim \mathcal{N}\left(\mathrm{Y}_{\mathrm{j}}, \Sigma\right)
\end{aligned}
$$$$
\text { where } i \neq j \text { for }\{H(t): t=1, \ldots, T\},
$$

where $Y_{i}$ is the mean feature vector for subject $i$, and $Y_{j}$ is the mean feature vector for subject $j$. The covariance matrix $\Sigma$ is assumed to be the same across subjects. Implicit in this formulation is the assumption that the $Y_{i} \neq Y_{j}$, whenever $i \neq j$, which is a necessary condition for ECG to provide a unique biometric signature. The distance between $Y_{i}$ and $Y_{j}$ sets the trade space for selecting ECG attributes and verification algorithms, as it affects the required number of heartbeats needed for making a decision whether the individual is an authentic user or an intruder.

To test the hypotheses $H 0$ and $H 1$, we calculate the log of the likelihood ratio statistic for whether the first $T$ heartbeats for subject $i$ come from the $j$ th subject. In the classical Neyman-Pearson formulation of hypothesis testing, $T$ would be fixed [47]. In the sequential procedure, we calculate the test statistic for values of $T$ until a decision is reached. Note that the verification methods depend on the Mahalanobis distance, and $Y$ is composed the 9-attribute feature vector. The test statistic as a function of $T$, the number of heartbeats is:

$$
S^{*}(T)=\sum_{t=1}^{T} \log \left(f_{1}(H(t))\right)-\log \left(f_{0}(H(t))\right)
$$

where

$$
\begin{aligned}
f_{0}(H(t))= & {[2 \pi]^{-K / 2}|\Sigma|^{-1 / 2} } \\
& \times \exp \left[-\frac{1}{2}\left(H(t)-Y_{i}\right)^{T} \Sigma^{-1}\left(H(t)-Y_{i}\right)\right], \\
f_{1}(H(t))= & {[2 \pi]^{-K / 2}|\Sigma|^{-1 / 2} } \\
& \times \exp \left[-\frac{1}{2}\left(H(t)-Y_{j}\right)^{T} \Sigma^{-1}\left(H(t)-Y_{j}\right)\right],
\end{aligned}
$$

where $K=9$ is the dimensionality of the vectors, and $Y_{j}$ is the mean for the alternative hypothesis. In principle, we would calculate the statistic $S^{*}(T)$ for each value of $T$, starting at $T=1$. For ECG analysis, at least two heartbeats are required. The features are the distances between fiducial points, normalized by the length of the heartbeat. This normalization insures that the verification procedure is tolerant to changes in overall heartrate attributable to varying physical, mental, or emotional state.

Computing $S^{*}(T)$ requires calculating (7) for each heartbeat, multiplying, and taking logs to compute the value defined in (6). Computationally, this can be simplified. The term $[2 \pi]^{-k / 2}|\Sigma|^{-1 / 2}$ is a constant that gets added and subtracted, so it can be ignored. The test procedure simplifies to calculate the quadratic forms (8) and (9):

$$
\begin{aligned}
& f_{0}(H(t)) \propto-\frac{1}{2}\left(H(t)-Y_{i}\right)^{T} \Sigma^{-1}\left(H(t)-Y_{i}\right), \\
& f_{1}(H(t)) \propto-\frac{1}{2}\left(H(t)-Y_{j}\right)^{T} \Sigma^{-1}\left(H(t)-Y_{j}\right) .
\end{aligned}
$$

Sum up values to compute $S^{*}(T)$ for each value of $T$, that is,

$$
S(T)=\sum_{t=1}^{T} \log \left[f_{1}(H(t))\right]-\log \left[f_{0}(H(t))\right] .
$$

The result of all this is a series of values for $S^{*}(T)$ for $T=1,2,3, \ldots$. Because the feature vector $H(t)$ depends on the estimated $R-R$ interval, a minimum of two heartbeats is needed. Thus, in practice, the "0th" heartbeat must be acquired, and $S^{*}(1)$ is computed from the 0 th and 1 st heartbeats and $S^{*}(T)$ for $T \geq 2$ are computed sequentially as each heartbeat is added to the sample. 
Comparing $S^{*}(T)$ to the critical values determines which hypothesis to accept. We define the errors $\alpha$ and $\beta$ as follows:

$$
\begin{aligned}
\alpha & =\operatorname{Pr}\{\text { Rejecting } H 0 \mid H 0 \text { is true }\} \\
& =\operatorname{Pr}\left\{S^{*}(T)>\log (B)\right\}, \\
\beta & =\operatorname{Pr}\{\text { Rejecting } H 1 \mid H 1 \text { is true }\} \\
& =\operatorname{Pr}\left\{S^{*}(T)<\log (A)\right\} .
\end{aligned}
$$

For a test of simple hypothesis, it has been shown [46] that (11)

$$
\begin{aligned}
& \log (A)=\log \left[\frac{\beta}{1-\alpha}\right], \\
& \log (B)=\log \left[\frac{1-\beta}{\alpha}\right] .
\end{aligned}
$$

To illustrate the application of the sequential procedure to the ECG signal, consider the example shown in Figure 8. Suppose the person presenting his/her credentials claims to be person $i$. Then the enrollment data for the $i$ th subject gives the estimated mean $Y_{i}$ under $H 0$. If the true identity is $j$, where $j \neq i$, then one could use $Y_{j}$ for the mean value under $H 1$. We consider 5 cases in which $H 0$ is false and the data come from five different individuals, labeled $1-5$ in Figure $8(a)$. In all cases, the test statistic quickly exceeds the decision threshold $\log (B)$ for $\alpha=\beta=0.01$. Comparing the behavior of the test statistics (Figure 8(a)) to the distance between the mean vector for the true identity and the mean vector for the declared identity (Figure 8(b)) reveals a direct correspondence. Note that these distances are computed from the training/enrollment data, while the test statistic depends on the enrolled means and the actual heartbeats observed in the test data. As one might expect, a large difference between the enrolled means for the true and declared identities corresponds to a large value of $S^{*}(T)$ and a rapid acceptance of $H 1$. When the true mean is close to the mean of the declared identity, $S^{*}(T)$ increases more slowly.

This leads to the final step in the formulation of the sequential procedure, namely, the selection of $i$ and $j$ for constructing the test statistic. The choice of $i$ is clear-it always corresponds to the declared identity of the individual presenting the credentials. To select $j$, we use the "closest imposter," that is, the enrolled individual with credentials closest to the declared individual. In other words, we select $j$ such that as

$$
\left\|Y_{i}-Y_{j}\right\|=\min \left\{k \ni k \neq i:\left\|Y_{i}-Y_{k}\right\|\right\},
$$

where $\left\|Y_{i}-Y_{j}\right\|$ is distance defined by

$$
\left\|Y_{i}-Y_{j}\right\|=\left(Y_{i}-Y_{j}\right)^{T} \Sigma^{-1}\left(Y_{i}-Y_{j}\right),
$$

and $\Sigma$ is the pooled covariance matrix. When $H 0$ is true, we use the nearest imposter to calculate the test statistic shown in Figure 8. The procedure determines that the $S^{*}(T)$ falls below the decision boundary, and $H 0$ is accepted.

\section{Results}

We present performance results for two data sets. The first data set, consisting of 29 subjects, was acquired under a strict protocol documented previously [1-4]. The second data set merges recordings from two data acquisitions discussed by Israel et al. [6]. Both datasets are single channel collections. Together, these data sets suggest the performance that can be expected for a moderate size population. In practice, however, a range of issues require further investigation: the effects of varying mental and emotional states on the ECG signal, the sensor placement and efficient data acquisition, generalization to larger populations, and the long-term stability of the ECG credentials. These issues are explored in the next section.

5.1. First Data Set. The ECG data analyzed in the work of Israel et al. [4] and Irvine et al. [5] provides a target performance for the sequential procedure. For this experiment, the single channel ECG data were collected at the base of the neck at a sampling rate of $1000 \mathrm{~Hz}$ with an 11-bit dynamic range. The population consisted of 29 males and females between the ages of 18 and 48, with no known cardiac anomalies. During each session, the subject's ECG was recorded while performing seven 2-minute tasks. The tasks were designed to elicit varying stress levels and to understand stress/recovery cycles. The results shown here used data from the subject's low stress tasks. The next section presents results for one of the high-stress tasks.

Setting the decision threshold based on $\alpha=\beta=0.01$, all 29 subjects were analyzed using the sequential procedure. When $H O$ is true, that is, the test data comes from the subject who is declared to be subject $i$, the results show that $H 0$ is accepted in all cases (Figure 9). We stopped processing at 15 heartbeats. In all cases, the decision was reached within that time span, and usually much sooner.

Similarly, when $H 1$ is true, the correct decision is generally reached in fewer than 15 heartbeats (Figure 10). In this set of results, the true identity for the test data is, in fact, the closest imposter. In only one case did the test procedure fail to reject an imposter within 15 heartbeats. In addition, we have computed the sequential tests when data for other subjects are used for the test set and the correct decision is always made in fewer heartbeats. Essentially, Figure 10 represents a worst case in which the subject trying to pose as someone else has a heartbeat that is fairly similar to the declared identity.

The sequential procedure performs well for the test data. An important practical issue is the number of heartbeats required to reach a decision. Figure 11 depicts the number of heartbeats required for a decision when $\mathrm{HO}$ is true (Figure 11, left side) and when $H 1$ is true (Figure 11, right side). In both cases, most of the individuals were identified using only 2 or 3 heartbeats. In cases where there is some ambiguity, however, additional heartbeats are needed to resolve the differences.

The number of heartbeats needed to reach a decision depends on the level of acceptable error. The results presented in Figures 9, 10 and 11 assume $\alpha=\beta=0.01$. 


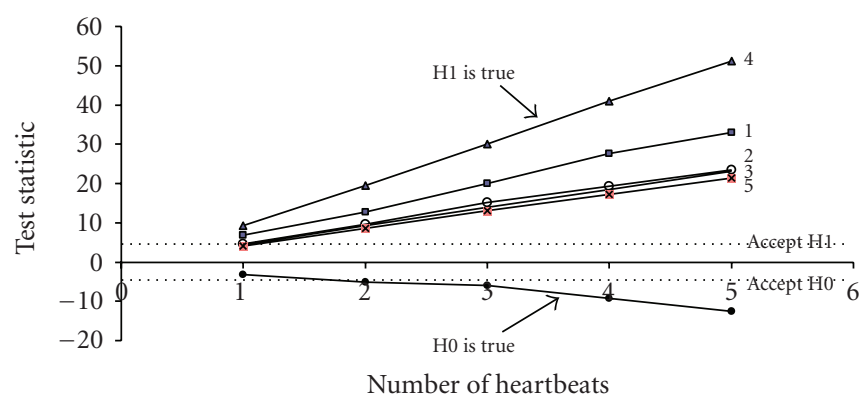

(a)

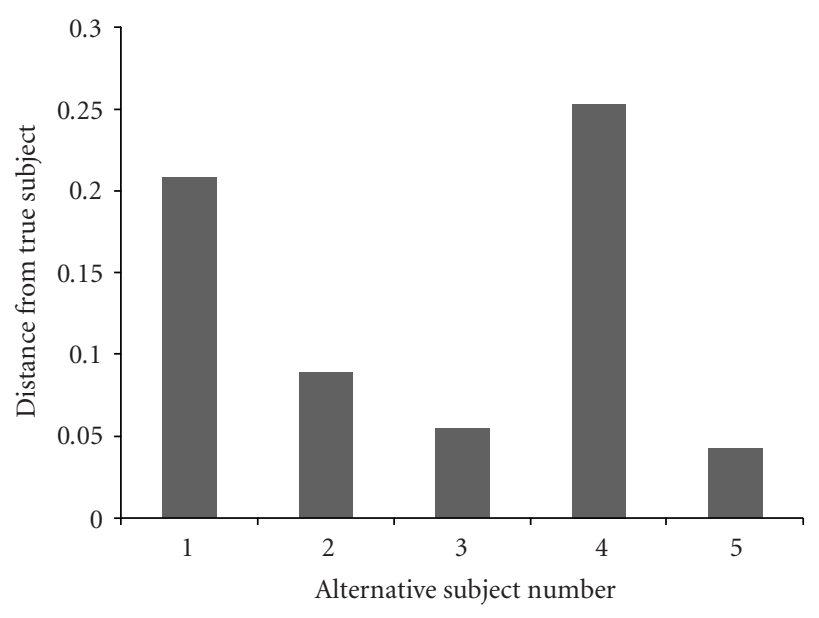

(b)

Figure 8: Example of a sequential procedure. (a) Sequential test statistic for a single declared identity when $H 0$ is true and for five imposters. (b) The distance of the declared identity to the five imposters.

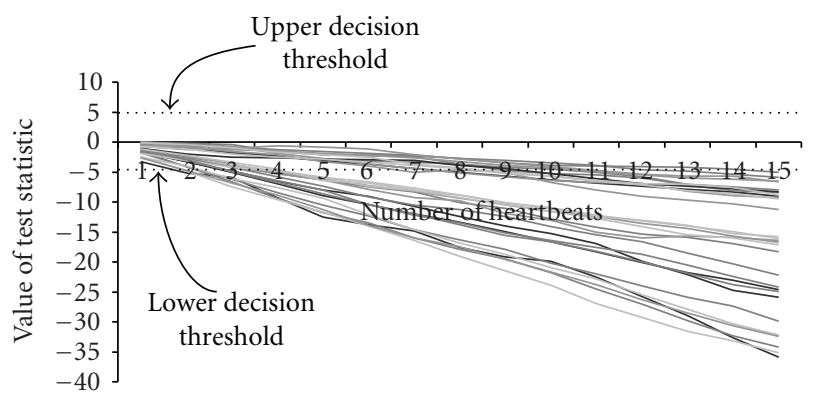

FIGURE 9: Sequential test statistics for all subjects when $H 0$ is true. The test data are from the declared individual.

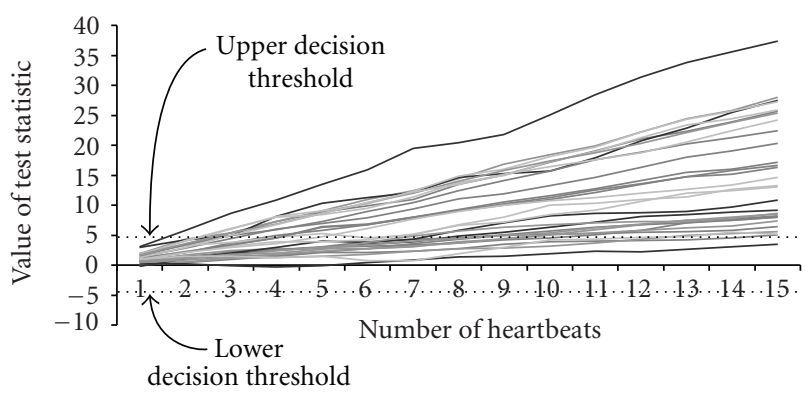

FIgURE 10: Sequential test statistics for all subjects when $H 1$ is true. The test data are from the subject closest to the declared individual, that is, the nearest imposter.

An inverse relationship exists between acceptable error rate and required number of heartbeats. Smaller levels of acceptable error will drive the decision process to require more data. Table 1 summarizes the performance for $\alpha=$ $\beta$ ranging from 0.1 to 0.0001 . More stringent constraints on $\alpha$ and $\beta$, for example, $\alpha=\beta=0.001$ or $\alpha=$ $\beta=0.0001$, generally require more heartbeats. As the acceptable error reduces, a decision is not always realized within 15 heartbeats. For the case of $\alpha=\beta=0.0001$, the procedure was run until a decision was reached for all subjects. When $H 0$ is true, the maximum number of heartbeats needed was 33 . When $H 1$ was true, the maximum was 37 heartbeats. In all cases, the correct decision was reached.

5.2. Second Data Set. Two additional ECG data collection campaigns used a simplified protocol and a standard, FDA approved ECG device. The clinical instrument recorded the ECG data at $256 \mathrm{~Hz}$ and quantized it to 7 bits. These data were acquired from two studies: one which collected single channel data from 28 subjects with the sensor placement at the wrist and one which collected single lead data from 47 subjects using a wearable sensor. The result is an additional 75 subjects.

The analysis followed the same procedure as with the first data set. Application of the sequential procedure for all 75 subjects was performed under both $H 0$ and $H 1$. Table 2 summarizes the results for the two cases $\alpha=\beta=0.05$ and $\alpha=\beta=0.01$, where the procedure ran for a maximum of 24 heartbeats. The results show that in a few instances a decision is not reached within the 24 heartbeats. For $\alpha=\beta=0.05$, when $H 0$ is true the procedure fails to decide for 2 subjects and 2 additional subjects are classified incorrectly. When $H 1$ is true, the procedure failed to decide for 1 subject and decided incorrectly for 1 subject.

A comparison of the results from the two data sets shows good consistency. A statistical comparison reveals no significant difference. Consider, for example, performance when $\alpha=\beta=0.05$. Under $H 0$, a statistical comparison of the correct acceptance rates yields a $t$-statistic of 1.39 . The corresponding $t$-statistic under $H 1$ is 0.58 . In short, performance for the two experiments is statistically indistinguishable. 


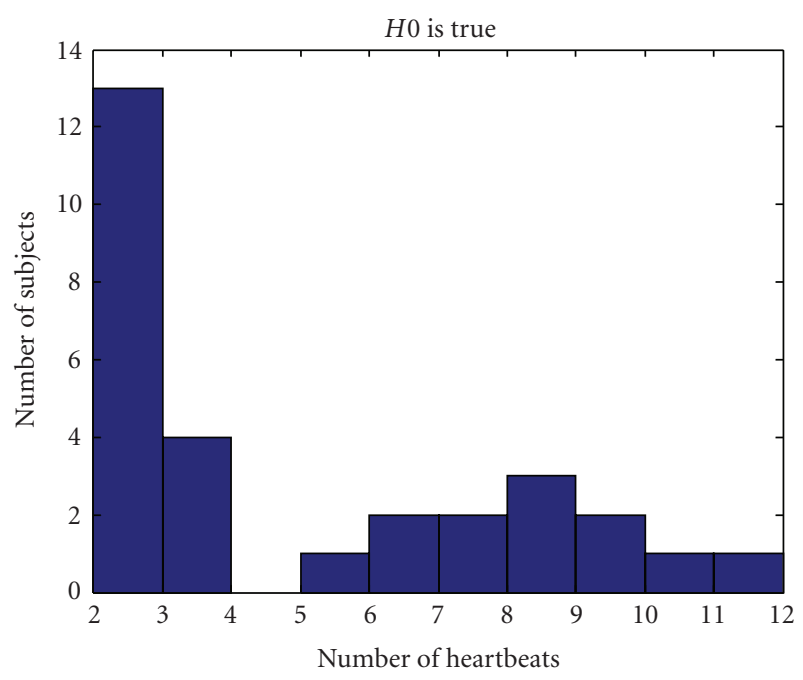

(a)

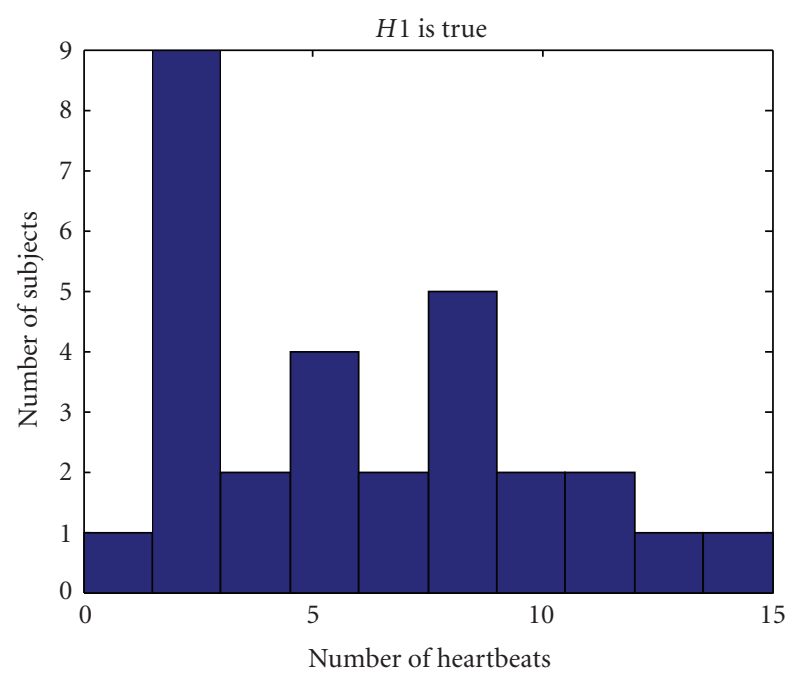

(b)

FIGURE 11: Histograms showing the number of heartbeats needed to reach a decision where the acceptable level of error is $\alpha=\beta=0.01$.

TABLE 1: Summary statistics for the number of heartbeats needed to reach a decision for varying levels of the acceptable error.

\begin{tabular}{lccccccccc}
\hline \multicolumn{3}{c}{ H0 is true } \\
$\begin{array}{l}\text { Allowable } \\
\text { error }(\alpha, \beta)\end{array}$ & $\begin{array}{c}\text { Mean no. } \\
\text { of } \\
\text { heartbeats }\end{array}$ & $\begin{array}{c}\text { Minimum } \\
\text { no. of } \\
\text { heartbeats }\end{array}$ & $\begin{array}{c}\text { Maximum } \\
\text { no. of } \\
\text { heartbeats }\end{array}$ & $\begin{array}{c}\text { Percent } \\
\text { resulting } \\
\text { in decision }\end{array}$ & $\begin{array}{c}\text { Allowable } \\
\text { error }(\alpha, \beta)\end{array}$ & $\begin{array}{c}\text { Mean no. } \\
\text { of } \\
\text { heartbeats }\end{array}$ & $\begin{array}{c}\text { Minimum } \\
\text { no. of } \\
\text { heartbeats }\end{array}$ & $\begin{array}{c}\text { Maximum } \\
\text { no. of } \\
\text { heartbeats }\end{array}$ & $\begin{array}{c}\text { Percent } \\
\text { resulting } \\
\text { in decision }\end{array}$ \\
\hline 0.1 & 3.38 & 2 & 8 & 100 & 0.1 & 3.655 & 2 & 11 & 100 \\
0.05 & 4.24 & 2 & 9 & 100 & 0.05 & 4.621 & 2 & 14 & 100 \\
0.01 & 6.07 & 2 & 15 & 100 & 0.01 & 6.500 & 2 & 15 \\
0.005 & 6.68 & 3 & 14 & 96.6 & 0.005 & 7.000 & 2 & 14 & 96.6 \\
0.001 & 7.28 & 3 & 13 & 86.2 & 0.001 & 7.792 & 3 & 15 \\
0.0005 & 7.96 & 4 & 15 & 86.2 & 0.0005 & 8.174 & 3 & 82.8 \\
0.0001 & 7.55 & 4 & 15 & 69.0 & 0.0001 & 7.647 & 4 & 15 & 14 \\
\hline
\end{tabular}

\section{Issues and Concerns}

The results presented in the previous section, while promising, were obtained from modest data sets collected under controlled conditions. To be operationally viable, a system must address performance across a range of conditions. Key issues to consider are

(i) heartrate variability, including changes in mental and emotional states,

(ii) sensor placement and data collection,

(iii) scalability to larger populations,

(iv) long-term viability of the ECG credentials.

Heartrate Variability. Heartrate, of course, varies with a person's mental or emotional state. Excitement or arousal from any number of stimuli can elevate the heartrate. Under the experimental protocol employed to collect the first data set, subjects performed a series of tasks designed to elicit varying mental and emotional states [1-4]. The subjects exhibited changes in heartrate associated with these

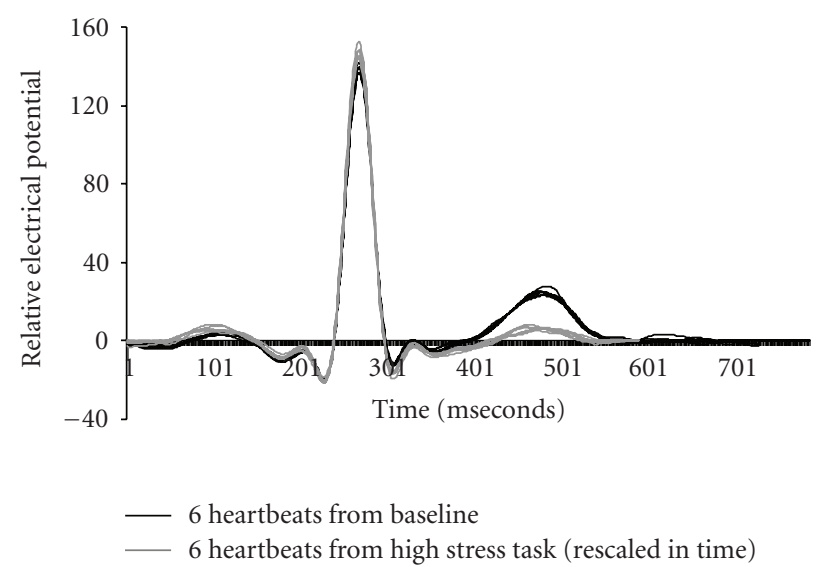

Figure 12: Aligned heartbeats from high stress and low stress tasks.

tasks. The fiducial features, however, show relatively small differences due to the variation in heartrate. To illustrate, consider Figure 12. For a single subject, Figure 12 presents 6 heartbeats from the baseline task in which the subject is 
TABLe 2: Analysis of second data set.

(a) Heartbeats required to reach a decision

\begin{tabular}{lccccccccc}
\hline \multicolumn{3}{c}{ H0 is true } \\
$\begin{array}{l}\text { Allowable } \\
\text { error }(\alpha, \beta)\end{array}$ & $\begin{array}{c}\text { Mean no. } \\
\text { of } \\
\text { heartbeats }\end{array}$ & $\begin{array}{c}\text { Minimum } \\
\text { no. of } \\
\text { heartbeats }\end{array}$ & $\begin{array}{c}\text { Maximum } \\
\text { no. of } \\
\text { heartbeats }\end{array}$ & $\begin{array}{c}\text { Percent } \\
\text { resulting } \\
\text { in decision }\end{array}$ & $\begin{array}{c}\text { Allowable } \\
\text { error }(\alpha, \beta)\end{array}$ & $\begin{array}{c}\text { Mean no. } \\
\text { of } \\
\text { heartbeats }\end{array}$ & $\begin{array}{c}\text { Minimum } \\
\text { no. of } \\
\text { heartbeats }\end{array}$ & $\begin{array}{c}\text { Maximum } \\
\text { no. of } \\
\text { heartbeats }\end{array}$ & $\begin{array}{c}\text { Percent } \\
\text { resulting } \\
\text { in decision }\end{array}$ \\
\hline 0.05 & 3.04 & 2 & 22 & 97.3 & 0.05 & 3.10 & 2 & 22 & 98.7 \\
0.01 & 4.93 & 2 & 24 & 92.0 & 0.01 & 4.99 & 2 & 24 & 92.0 \\
\hline
\end{tabular}

(b) Correct decision rates

\begin{tabular}{lccc}
\hline & $H 0$ is true & & $H 1$ is true \\
\hline $\begin{array}{l}\text { Allowable error } \\
(\alpha, \beta)\end{array}$ & $\begin{array}{c}\text { Percent resulting in } \\
\text { correct decision }\end{array}$ & $\begin{array}{c}\text { Allowable error } \\
(\alpha, \beta)\end{array}$ & $\begin{array}{c}\text { Percent resulting in } \\
\text { correct decision }\end{array}$ \\
\hline 0.05 & 94.7 & 0.05 & 97.3 \\
0.01 & 89.3 & 0.01 & 90.7 \\
\hline
\end{tabular}

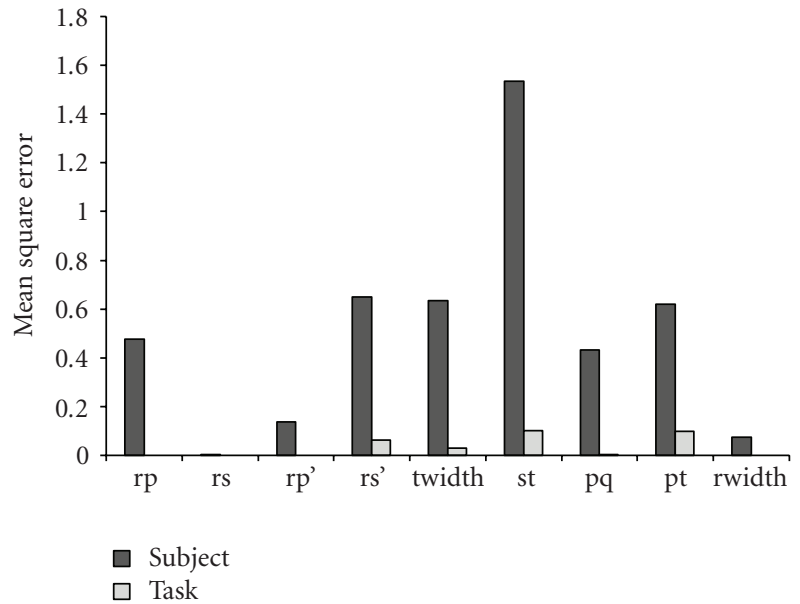

FIGURE 13: Comparison of variance attributable to subject and task.

seated at rest. In addition, 6 heartbeats from a high stress task (a virtual reality driving simulation) were temporally rescaled and overlaid on the same graph. For this particular subject, the mean $R-R$ interval for the baseline task was 0.715 seconds and for the high stress task it was 0.580 seconds. However, by a linear rescaling, the high-stress heartbeats align well with the baseline heartbeats. A difference in the height of the $T$ wave is evident but the fiducial features depend on the relative positions of the peaks, not the heights.

Delving deeper than the visual evidence for a single subject, we conducted a systematic analysis of the sources of variance in the fiducial features using a multivariate analysis of variance (MANOVA). The 29 subjects performed all seven tasks in the experimental protocol eliciting a range of stimulation. The MANOVA shows that there are small, but statistically significant, differences in the fiducials across the various tasks, indicating that there are subtle differences in the ECG signal that are more complex than a linear rescaling. This source of variance, however, is typically one or two orders of magnitude smaller than the variance across subjects. Figure 13 shows the relationships between the two mean square errors for each fiducial, and the variation across subjects is far more pronounced than the variation due to task. This relationship is why the fiducial-based features are likely to provide good information about a subject's identity across a range of conditions.

To verify this hypothesis, we explored the effect of varying the level of arousal of the subject. The protocol used for collecting Dataset 1 included a set of tasks designed to elicit varying levels of stimulation or arousal [1-4]. Using the baseline, low stress task for training, we processed data from one of the high-stress tasks for testing. Specifically, the subjects performed an arithmetic task designed to affect both stress and cognitive loads. The effectiveness of the task is evident in that the mean $R-R$ interval decreased from a baseline of 0.83 to 0.76 for this task. Nevertheless, the sequential procedure yielded good performance on these data (Table 3).

If alternative attributes are evaluated in the trade space, such as wavelets [35] or Legendre coefficients [48], then their sensitivity must also be evaluated in the same manner as above. Likewise, incorporating other verification algorithms such as PCA [5, 49] or Gaussian modeling [50] will require substituting their characteristics into the sequential process. Regardless, the minimum number of heartbeats is appropriate for comparing systems.

Sensor Placement. Dataset 1 collected ECG traces from the base of the neck. Dataset 2 collected ECG traces on the forearms. Both collections used medical quality single use electrodes. However, any operational system must design a more robust collection method. This method must have reusable electrodes, a concept of employment for locating electrodes on normally exposed skin, and other human factors. These issues are outside the scope of this paper. However, the concept of employment does raise significant concerns about the noise floor for an operational system. As the noise floor increases the separability between the subject and the nearest imposter reduces. 
TABLE 3: Effects of varying levels of stimulation.

\begin{tabular}{|c|c|c|c|c|c|c|c|c|c|}
\hline \multicolumn{5}{|c|}{$\mathrm{H} 0$ is true } & \multicolumn{5}{|c|}{$\mathrm{H} 1$ is true } \\
\hline $\begin{array}{l}\text { Allowable } \\
\text { error }(\alpha, \beta)\end{array}$ & $\begin{array}{l}\text { Mean no. } \\
\text { of } \\
\text { heartbeats }\end{array}$ & $\begin{array}{c}\text { Minimum } \\
\text { no. of } \\
\text { heartbeats }\end{array}$ & $\begin{array}{c}\text { Maximum } \\
\text { no. of } \\
\text { heartbeats }\end{array}$ & $\begin{array}{c}\text { Percent } \\
\text { correct } \\
\text { decision }\end{array}$ & $\begin{array}{l}\text { Allowable } \\
\text { error }(\alpha, \beta)\end{array}$ & $\begin{array}{l}\text { Mean no. } \\
\text { of } \\
\text { heartbeats }\end{array}$ & $\begin{array}{c}\text { Minimum } \\
\text { no. of } \\
\text { heartbeats }\end{array}$ & $\begin{array}{c}\text { Maximum } \\
\text { no. of } \\
\text { heartbeats }\end{array}$ & $\begin{array}{l}\text { Percent } \\
\text { correct } \\
\text { decision }\end{array}$ \\
\hline 0.01 & 5.57 & 2 & 17 & 96.6 & 0.01 & 4.41 & 2 & 10 & 93.1 \\
\hline
\end{tabular}

Scalability. Depending on the application, an ECG-based identity verification system may need to store credentials for hundreds or thousands of individuals. The recent experiments lack the sample sizes needed to determine large-scale performance, and the next step is to assess performance over much larger data sets. Because our approach compares the credentials for the declared subject to the nearest imposter, the separability among members of the training set is critical. By always choosing $j$ to be the closest imposter, we guard against accepting a person's credentials too readily. Fortunately, determining the closest imposter is performed using training data, offline, which greatly improves the processing efficiency and system usability. It does, however, raise a concern about extending these methods to applications involving large enrolled populations. An alternative approach is to select $j$ based on the features extracted from the first heartbeat. One could select $j$ to be the member of the enrollment set closest to the first heartbeat from the test data, where $j \neq i$. In terms of the statistical formulation, $H 1$ is no longer a simple hypothesis, since $j$ is chosen to minimize a criterion over the full enrollment set. A simple experiment on a subset of the data revealed mean decision times of approximately 8 heartbeats for $\alpha=\beta=0.01$, compared to 6.5 using the nearest imposter. Further investigation of this issue is still needed.

Long-Term Viability. Characteristics of an individual's ECG can change for a variety of medical reasons, including cardiovascular disease and changes in medication. Research has examined these issues from a clinical perspective, but further investigation is needed to understand how these factors affect ECG as a biometric for identification. For the data analyzed in this paper, the time difference between the training and test sets ranged from minutes to months, but no truly longterm differences have been studied. Such a study needs to be conducted, and existing clinical measurements are likely to be the most readily available source of data. Depending on the concept of employment, however, periodic re-enrollment may be one strategy for addressing long-term changes in an individual's ECG signal.

\section{Discussion}

This research builds on previous investigations into the viability of ECG as a biometric for human identification. We focus specifically on a procedure for exploiting the ECG signal for identity verification, with the optimization metric being the number of heartbeats needed for the system to make a decision. By using a method based on a sequential procedure for statistical hypothesis testing, data acquisition time is minimized. For the two data sets analyzed here, the approach generally yields the correct decision given enough heartbeats.

For modest levels of acceptable risk $(\alpha=\beta=0.01$ or 0.05 ), the decision is often made after only 3 or 4 heartbeats and is almost always made within 15 heartbeats. In practice, this implies a data acquisition time of approximately 5 to 15 seconds. Lower risk tolerance (e.g., $\alpha=\beta=0.0001$ ) could require 30 seconds or more to reach a decision for some individuals.

Whether the data acquisition time is acceptable in practice will depend, of course, on the specific application. One attractive approach is to use ECG in conjunction with other biometrics, such as fingerprint and hand geometry. This multimodality approach could support less demanding performance limits for the ECG (e.g., $\alpha=\beta=0.01$ ), while providing a high level of security that will be very difficult to forge or circumvent.

Further investigations are still needed to refine and validate the methods presented here. Specific avenues for future research include the following.

(i) Assessment of performance over a much larger population of test subjects: larger data sets, including data collected at greater time intervals, are necessary to characterize the behavior of these methods.

(ii) Investigation of robustness to physical, mental, and emotional states and longer baselines between visits: heartrate will vary with a variety of stimuli. Irvine et al. [3] and Israel et al. [4] demonstrated that with proper normalization, the fiducial-based features are robust to mental and emotional states. Further validation that similar results hold for the sequential procedures would be useful.

(iii) Exploration of alternative feature extraction methods and verification algorithms: researchers have proposed a variety of alternative feature extraction methods, including variations on the fiducial features, principal component analysis, template matching, and frequency-domain approaches. These methods can be integrated into the sequential procedure framework, and a comparison of different approaches could prove enlightening.

\section{Acknowledgments}

This research was sponsored by the Defense Advanced Research Projects Agency (DARPA) under Contract no. DABT63-00-C-1039, with additional support from Charles Stark Draper Laboratory and SAIC. The data were collected 
at the Virtual Reality Medical Center under the supervision of Drs. Mark and Brenda Wiederhold. Additional assistance was provided by Dr. Rodney Meyer, Dr. Lauren Gavshon, Ms. Shannon McGee, and Ms. Elizabeth Rosenfeld. The authors also wish to thank Dr. P. Jonathon Phillips, formerly of DARPA, for valuable comments concerning the development of this work. Finally, the authors wish to thank the anonymous reviewers for valuable insights and suggestions. The views expressed here are those of the authors and do not necessarily represent the positions of DARPA, SAIC, Draper Laboratory, or VRMC.

\section{References}

[1] J. M. Irvine, B. K. Wiederhold, L. W. Gavshon, et al., "Heart rate variability: a new biometric for human identification," in Proceedings of the International Conference on Artificial Intelligence (IC-AI '01), pp. 1106-1111, Las Vegas, Nev, USA, June 2001.

[2] J. M. Irvine, S. A. Israel, A. Cheng, M. D. Wiederhold, B. K. Wiederhold, and S. McGehee, "Validation of new biometrics for human identification," in Proceedings of the Joint Statistical Meetings (JSM '02), New York, NY, USA, August 2002.

[3] J. M. Irvine, S. A. Israel, M. D. Wiederhold, and B. K. Wiederhold, "A new biometric: human identification from circulatory function," in Proceedings of the Joint Statistical Meetings of the American Statistical Association, pp. 1-7, San Francisco, Calif, USA, August 2003.

[4] S. A. Israel, J. M. Irvine, A. Cheng, M. D. Wiederhold, and B. K. Wiederhold, "ECG to identify individuals," Pattern Recognition, vol. 38, no. 1, pp. 133-142, 2005.

[5] J. M. Irvine, S. A. Israel, W. Todd Scruggs, and W. J. Worek, "eigenPulse: robust human identification from cardiovascular function," Pattern Recognition, vol. 41, no. 11, pp. 3427-3435, 2008.

[6] S. A. Israel, J. M. Irvine, B. K. Wiederhold, and M. D. Wiederhold, "The heartbeat: the living biometric," in Biometrics: Theory, Methods, and Applications, N. V. Boulgouris, E. Micheli-Tzanakou, and K. N. Plataniotis, Eds., Wiley-IEEE Press, New York, NY, USA, 2009.

[7] M. D. Wiederhold, S. A. Israel, R. P. Meyer, and J. M. Irvine, Human Identification by Analysis of Physiometric Variation, SAIC, San Diego, Calif, USA, 2006.

[8] L. Biel, O. Pettersson, L. Philipson, and P. Wide, "ECG analysis: a new approach in human identification," IEEE Transactions on Instrumentation and Measurement, vol. 50, no. 3, pp. 808812, 2001.

[9] Y. Wang, F. Agrafioti, D. Hatzinakos, and K. Plataniotis, "Analysis of human electrocardiogram for biometric recognition," EURASIP Journal on Advances in Signal Processing, vol. 2008, Article ID 148658, 11 pages, 2008.

[10] T. W. Shen, W. J. Tompkins, and Y. H. Hu, "One-lead ECG for identity verification," in Proceedings of the 2nd Joint Conference of the IEEE Engineering in Medicine and Biology Society and the 24th Annual Conference and the Annual Fall Meeting of the Biomedical Engineering Society (EMBS/BMES '02), vol. 1, pp. 62-63, Houston, Tex, USA, October 2002.

[11] F. R. Yu, H. Tang, V. C. M. Leung, J. Liu, and C.-H. Lung, "Biometric-based user authentication in mobile ad hoc networks," Security and Communication Networks, vol. 1, no. 1, pp. 5-16, 2008.
[12] R. Palaniappan and S. M. Krishnan, "Identifying individuals using ECG beats," in Proceedings of the International Conference on Signal Processing and Communications (SPCOM '04), pp. 569-572, Bangalore, India, December 2004.

[13] M. Kyoso and A. Uchiyama, "Development of an ECG identification system," in Proceedings of the 23rd Annual International Conference of the IEEE Engineering in Medicine and Biology Society (EMBS '01), vol. 4, pp. 3721-3723, Istanbul, Turkey, October 2001.

[14] G. Wubbeler, M. Stavridis, D. Kreiseler, R.-D. Bousseljot, and C. Elster, "Verification of humans using the electrocardiogram," Pattern Recognition Letters, vol. 28, no. 10, pp. 11721175, 2007.

[15] G. D. Clifford, F. Azuaje, and P. E. McSharry, Advanced Methods and Tools for ECG Data Analysis, Artech House, Norwood, Mass, USA, 2006.

[16] H. C. Bazett, "An analysis of time relations of electrocardiograms," Heart, vol. 7, pp. 353-370, 1920.

[17] D. P. Golden Jr., R. A. Wolthuis, and G. W. Hoffler, "A spectral analysis of the normal resting electrocardiogram," IEEE Transactions on Biomedical Engineering, vol. 20, no. 5, pp. 366-372, 1973.

[18] D. Hawkins, "Body of evidence," U.S. News \& World Report, vol. 132, no. 5, pp. 60-62, 2002.

[19] M. P. S. Chawla, H. K. Verma, and V. Kumar, "A new statistical PCA-ICA algorithm for location of R-peaks in ECG," International Journal of Cardiology, vol. 129, no. 1, pp. 146148, 2008.

[20] P. de Chazal, C. Heneghan, E. Sheridan, R. Reilly, P. Nolan, and M. O'Malley, “Automated processing of the singlelead electrocardiogram for the detection of obstructive sleep apnoea," IEEE Transactions on Biomedical Engineering, vol. 50, no. 6, pp. 686-696, 2003.

[21] M. Kotas, "Robust projective filtering of time-warped ECG beats," Computer Methods and Programs in Biomedicine, vol. 92, no. 2, pp. 161-172, 2008.

[22] K. Noponen, J. Kortelainen, and T. Seppänen, "Invariant trajectory classification of dynamical systems with a case study on ECG," Pattern Recognition, vol. 42, no. 9, pp. 1832-1844, 2009.

[23] R. Nygaard, G. Melnikov, and A. K. Katsaggelos, "A rate distortion optimal ECG coding algorithm," IEEE Transactions on Biomedical Engineering, vol. 48, no. 1, pp. 28-40, 2001.

[24] N. C. Oza and K. Tumer, "Classifier ensembles: select realworld applications," Information Fusion, vol. 9, no. 1, pp. 4-20, 2008.

[25] R. Palaniappan and K. V. R. Ravi, "Improving visual evoked potential feature classification for person recognition using PCA and normalization," Pattern Recognition Letters, vol. 27, no. 7, pp. 726-733, 2006.

[26] K. N. Plataniotis, D. Hatzinakos, and J. K. M. Lee, "ECG biometric recognition without fiducial detection," in Proceedings of the Biometric Consortium Conference (BCC '06), pp. 1-6, Baltimore, Md, USA, September 2006.

[27] A. van Oosterom, R. Hoekema, and G. J. H. Uijen, "Geometrical factors affecting the interindividual variability of the ECG and the VCG," Journal of Electrocardiology, vol. 33, no. 3, pp. 219-227, 2000.

[28] M. Turk and A. Pentland, "Eigenfaces for recognition," Journal of Cognitive Neuroscience, vol. 3, no. 1, pp. 71-86, 1991.

[29] F. M. Bui, F. Agrafioti, and D. Hatzinakos, "Electrocardiogram biometric," in Biometrics: Theory, Methods, and Applications, N. V. Boulgouris, E. Micheli-Tzanakou, and K. N. Plataniotis, Eds., Wiley-IEEE Press, New York, NY, USA, 2009. 
[30] F. Agrafioti and D. Hatzinakos, "ECG biometric analysis in cardiac irregularity conditions," Signal, Image and Video Processing, pp. 1683-1703, 2008.

[31] D. Dubin, Rapid Interpretation of ECGs, Cover, Tampa, Fla, USA, 6th edition, 2000.

[32] E. N. Marieb, Essential of Human Anatomy and Physiology, Benjamin Cummings, San Francisco, Calif, USA, 7th edition, 2003.

[33] T. Pawar, N. S. Anantakrishnan, S. Chaudhuri, and S. P. Duttagupta, "Transition detection in body movement activities for wearable ECG," IEEE Transactions on Biomedical Engineering, vol. 54, no. 6, pp. 1149-1152, 2007.

[34] R. Jane, H. Rix, P. Caminal, and P. Laguna, "Alignment methods for averaging of high-resolution cardiac signals: a comparative study of performance," IEEE Transactions on Biomedical Engineering, vol. 38, no. 6, pp. 571-579, 1991.

[35] P. Laguna, R. Jane, O. Meste, et al., "Adaptive filter for eventrelated bioelectric signals using an impulse correlated reference input: comparison with signal averaging techniques," IEEE Transactions on Biomedical Engineering, vol. 39, no. 10, pp. 1032-1044, 1992.

[36] M. Alfaouri and K. Daqrouq, "ECG signal denoising by wavelet transform thresholding," American Journal of Applied Sciences, vol. 5, no. 3, pp. 276-281, 2008.

[37] W. Zhang, X. Wang, L. Ge, and Z. Zhang, "Noise reduction in ECG signal based on adaptive wavelet transform," in Proceedings of the 27th Annual International Conference of the IEEE Engineering in Medicine and Biology Society (EMBS '05), pp. 2699-2702, Shanghai, China, January 2005.

[38] V. Almenar and A. Albiol, "A new adaptive scheme for ECG enhancement," Signal Processing, vol. 75, no. 3, pp. 253-263, 1999.

[39] M. Elena, J. M. Quero, and I. Borrego, "An optimal technique for ECG noise reduction in real time applications," in Proceedings of the Annual International Conference on Computers in Cardiology (CIC '06), vol. 33, pp. 225-228, Valencia, Spain, September 2006.

[40] A. K. Barros, M. Yoshizawa, and Y. Yasuda, "Filtering noncorrelated noise in impedance cardiography," IEEE Transactions on Biomedical Engineering, vol. 42, no. 3, pp. 324-327, 1995.

[41] A. K. Barros and N. Ohnishi, "Heart instantaneous frequency (HIF): an alternative approach to extract heart rate variability," IEEE Transactions on Biomedical Engineering, vol. 48, no. 8, pp. 850-855, 2001.

[42] P. S. Hamilton, "A comparison of adaptive and nonadaptive filters for reduction of power line interference in the ECG," IEEE Transactions on Biomedical Engineering, vol. 43, no. 1, pp. 105-109, 1996.

[43] A. H. Al-Khalidi, M. E. Lewis, J. N. Townened, R. S. Bonser, and J. H. Coote, "A novel and simple technique to allow detection of the position of the R-waves from intraventricular pressure waveforms: application to the conductance catheter method," IEEE Transactions on Biomedical Engineering, vol. 48, no. 5, pp. 606-610, 2001.

[44] B.-U. Kohler, C. Hennig, and R. Orglmeister, "The principles of software QRS detection," IEEE Engineering in Medicine and Biology Magazine, vol. 21, no. 1, pp. 42-57, 2002.

[45] M. Ghosh, Handbook of Sequential Analysis, CRC Press, Boca Raton, Fla, USA, 1st edition, 1991.

[46] A. Wald, Sequential Analysis, Dover, New York, NY, USA, 1994.

[47] E. L. Lehmann and J. P. Romano, Testing Statistical Hypotheses, Springer, New York, NY, USA, 3rd edition, 2008.
[48] J. Faganeli and F. Jager, "Automatic distinguishing between ischemic and heart-rate related transient ST segment episodes in ambulatory ECG records," in Proceedings of the 35th Annual International Conference on Computers in Cardiology (CIC '08), vol. 35, pp. 381-384, Bologna, Italy, September 2008.

[49] G. B. Moody and R. G. Mark, "QRS morphology representation and noise estimation using the Karhunen-Loeve transform," in Proceedings of the Annual International Conference on Computers in Cardiology (CIC '89), vol. 16, pp. 269-272, Jerusalem, Israel, September 1989.

[50] D. Clifford, A. Shoeb, P. E. McSharry, and B. A. Janz, "Modelbased filtering, compression and classification of the ECG," International Journal of Bioelectromagnetism, vol. 7, no. 1, pp. 158-161, 2005. 